\title{
APPROXIMATING ZEROS OF ACCRETIVE OPERATORS
}

\author{
SIMEON REICH
}

ABSTRACT. Let $A$ be an $m$-accretive set in a reflexive Banach space $E$ with a Gateaux differentiable norm. For positive $r$ let $J_{r}$ denote the resolvent of $A$. If the duality mapping of $E$ is weakly sequentially continuous and 0 is in the range of $A$, then for each $x$ in $E$ the strong $\lim _{r \rightarrow \infty} J_{r} x$ exists and belongs to $A^{-1}(0)$. This is an extension to a Banach space setting of a result previously known only for Hilbert space.

Let $H$ be a real Hilbert space and $U \subset H \times H$ a maximal monotone operator. For each positive $r$ there is a unique $y_{r}$ in $H$ such that $0 \in y_{r}+r U\left(y_{r}\right)$. It is known [4] that if 0 belongs to the range of $U$, then the strong $\lim _{r \rightarrow \infty} y_{r}$ exists and is the point of $U^{-1}(0)$ closest to 0 . It is our purpose in this note to extend this result to accretive operators in certain Banach spaces. According to [4], this leads to the possibility of calculating a zero of the given operator as the limit of an iteratively constructed sequence. Our method of proof is not a direct generalization of the Hilbert space proof. It works, however, only in a restricted class of Banach spaces. The question of whether our theorem is valid in other Banach spaces remains open.

Let $E^{*}$ denote the dual of a real Banach space $E$. The duality mapping $J$ from $E$ into the family of nonempty subsets of $E^{*}$ is defined by

$$
J(x)=\left\{x^{*} \in E^{*}:\left(x, x^{*}\right)=\|x\|^{2} \text { and }\left\|x^{*}\right\|=\|x\|\right\} \text {. }
$$

$J$ is single-valued if and only if the norm of $E$ is Gâteaux differentiable. If $A$ is a subset of $E \times E$ and $x \in E$, we define

$$
A x=\{y \in E:[x, y] \in A\}
$$

and set

$$
D(A)=\{x \in E: A x \neq \varnothing\} .
$$

The range of $A$ is defined by

$$
R(A)=\bigcup\{A x: x \in D(A)\}
$$

Received by the editors October 1, 1974.

AMS (MOS) subject clas sifications (1970). Primary 47H15, 47H05; Secondary 47B44, $40 \mathrm{~A} 05$.

Key words and phrases. Accretive, duality mapping, nonexpansive retract, strong and weak convergence. 
and its inverse by

$$
A^{-1} y=\{x \in E: y \in A x\} \text {. }
$$

$I$ will stand for the identity operator on $E$. The closure of a subset $D$ of $E$ will be denoted by $\mathrm{cl}(D)$. A mapping $T: D \rightarrow E$ is said to be nonexpansive if $\|T x-T y\| \leq\|x-y\|$ for all $x$ and $y$ in $D$. In the sequel, $\rightarrow$ and $\rightarrow$ will denote strong and weak convergence respectively.

A subset $A$ of $E \times E$ is called accretive [7] if for all $x_{i} \in D(A)$ and $y_{i} \in A x_{i}, i=1,2$, there exists $j \in J\left(x_{1}-x_{2}\right)$ such that $\left(y_{1}-y_{2}, j\right) \geq 0$. Let $D$ be a subset of $E$ and $A$ an accretive set (=accretive operator) with $D(A) \subset D . A$ is said to be maximal accretive in $D$ if there is no proper accretive extension $B$ of $A$ with $D(B) \subset D$. An accretive set $A$ is maximal accretive if it is maximal accretive in $E$. It is $m$-accretive if $R(I+A)=E$. (It follows that $R(I+r A)=E$ for all positive $r_{\text {. }}$ ) If $T: E \rightarrow E$ is nonexpansive, then $I-T$ is $m$-accretive. If $A$ is $m$-accretive, then it is maximal accretive, but the converse is not true in general. If $A$ is accretive one can define, for each $r>0$, a nonexpansive single-valued mapping $J_{r}: R(I+r A)$ $\rightarrow D(A)$ by $J_{r}=(I+r A)^{-1}$. It is called the resolvent of $A$. Conditions which imply that an $m$-accretive set is surjective can be found in [10].

The duality mapping $J$ of a Banach space $E$ with a Gâteaux differentiable norm [5] is said to be weakly sequentially continuous if $x_{n}-x$ in $E$ implies that $\left\{J\left(x_{n}\right)\right\}$ converges weak star to $J(x)$ in $E^{*}$. This happens, for example, if $E$ is a Hilbert space, or finite-dimensional and smooth, or $l^{p}$, $1<p<\infty$. This property of Banach spaces was introduced by Browder [1]. More information can be found in [6].

Lemma. Let $A$ be a maximal accretive set in a Banach space $E$ whose norm is Gâteaux differentiable. Let $x_{n} \in D(A), y_{n} \in A x_{n}, x_{n} \rightarrow x$, and $y_{n} \rightarrow y$. If the duality mapping $J$ is weakly sequentially continuous, then $[x, y] \epsilon A$.

Proof. Let $z \in D(A)$ and $w \in A z$. We have

$$
\begin{aligned}
& \left|\left(y_{n}-w, J\left(x_{n}-z\right)\right)-(y-w, J(x-z))\right| \\
& \quad \leq\left|\left(y_{n}-y, J\left(x_{n}-z\right)\right)\right|+\left|\left(y-w, J\left(x_{n}-z\right)-J(x-z)\right)\right| \\
& \quad \leq\left\|y_{n}-y\right\|\left\|x_{n}-z\right\|+\left|\left(y-w, J\left(x_{n}-z\right)-J(x-z)\right)\right| .
\end{aligned}
$$

Thus

$$
(y-w, J(x-z))=\lim _{n \rightarrow \infty}\left(y_{n}-w, J\left(x_{n}-z\right)\right) \geq 0 .
$$

The result follows. 
A closed subset $C$ of a Banach space $E$ is called a nonexpansive retract of $E$ if there exists a retraction of $E$ onto $C$ which is a nonexpansive mapping. A retraction $P: E \rightarrow C$ is called a sunny retraction if $P(x)=v$ implies that $P(v+r(x-v))=v$ for all $x \in E$ and $r \geq 0$. (We prefer this term to the one used by Bruck [3] because suns already occur in approximation theory.) If there exists a retraction $P: E \rightarrow C$ which is both sunny and nonexpansive, then $C$ is said to be a sunny nonexpansive retract of $E$. If $C$ is a sunny nonexpansive retract of a Banach space whose norm is Gâteaux differentiable, then the sunny nonexpansive retraction on $C$ is unique [3, Theorem 1], [8, Lemma 2.7]. The metric projection on a closed and convex subset of a Hilbert space is both sunny and nonexpansive.

Theorem. Let $A$ be an m-accretive set in a reflexive Banach space $E$ with a Gâteaux differentiable norm. If the duality mapping $J$ of $E$ is weakly sequentially continuous and $0 \in R(A)$, then for each $x$ in $E$ the strong $\lim _{r \rightarrow \infty} J_{r} x$ exists and belongs to $A^{-1}(0)$.

Proof. Let the positive sequence $\left\{r_{n}: n=1,2, \ldots\right\}$ tend to infinity. Let $x \in E$ and $y \in A^{-1}(0)$. Set $x_{n}=J_{r_{n}} x$. We have $\left(x_{n}-x, J\left(y-x_{n}\right)\right) \geq 0$ because $\left(x-x_{n}\right) / r_{n}$ belongs to $A x_{n}$ and 0 belongs to $A y$. Consequently,

$$
\left\|y-x_{n}\right\|^{2} \leq\left(y-x, J\left(y-x_{n}\right)\right) \leq\|y-x\|\left\|y-x_{n}\right\|
$$

and $\left\{x_{n}\right\}$ is bounded. Let $P x$ be the weak limit of a subsequence $\left\{x_{k}\right\}$ of $\left\{x_{n}\right\}$. Clearly $\left(x-x_{k}\right) / r_{k} \rightarrow 0$. By the Lemma, $[P x, 0]$ belongs to $A$. Therefore

$$
\left\|P x-x_{k}\right\|^{2} \leq\left(P x-x, J\left(P x-x_{k}\right)\right) \rightarrow 0 .
$$

Thus $\left\{x_{k}\right\}$ converges strongly to $P x$. It follows that $(P x-x, J(y-P x)) \geq 0$ for all $x$ in $E$ and $y$ in $A^{-1}(0)$. In other words [8, Lemma 2.7], $P: E \rightarrow$ $A^{-1}(0)$ is both sunny and nonexpansive. Since $P$ is necessarily unique, the sequence $\left\{x_{n}\right\}$ itself converges strongly to $P x$. This completes the proof.

Corollary (cf. [8, Theorem 3.2]). Let $T$ be a nonexpansive self-mapping of $E$, a reflexive Banach space with a Gâteaux differentiable norm. Suppose that $T$ has a nonempty fixed point set and that $E$ has a weakly sequentially continuous duality mapping. Let $x$ belong to E. For each $0<k<1$ let $x_{k}$ satisfy $x_{k}=k T x_{k}+(1-k) x$. Then the strong $\lim _{k \rightarrow 1-} x_{k}$ exists and is $a$ fixed point of $T$.

In the course of the proof of the Theorem it has been established that $A^{-1}(0)$ is a nonexpansive retract of $E$. Since $A^{-1}(0)$ is the fixed point set 
of the nonexpansive mapping $J_{r}$ (for all $r>0$ ), this is also a consequence of [2, Theorem 2]. In a similar setting, $\mathrm{cl}(D(A))$ is also a nonexpansive retract of $E$ [9, Theorem 3.7].

Remark. A version of the Theorem is true for a certain class of accretive operators which are not necessarily $m$-accretive.

Acknowledgement. I am grateful to Professor Ronald E. Bruck, Jr. for kindly providing me with a preprint of [4].

\section{REFERENCES}

1. F. E. Browder, Fixed point theorems for nonlinear semicontractive mappings in Banach spaces, Arch. Rational Mech. Anal. 21 (1965/66), 259-269. MR 34 \#641.

2. R. E. Bruck, Jr., Properties of fixed point sets of nonexpansive mappings in Banach spaces, Trans. Amer. Math. Soc. 179 (1973), 251-262.

3. - Nonexpansive projections on subsets of Banach spaces, Pacific J. Math. 47. (1973), 341-355.

4. - A strongly convergent iterative solution of $0 \epsilon U(x)$ for a maximal monotone operator U in Hilbert space, J. Math. Anal. Appl. 48 (1974), 114-126.

5. J.-P. Gossez, A note on multivalued monotone operators, Michigan Math. J. 17 (1970), 347-350. MR $43 \# 7978$.

6. J.-P. Gossez and E. Lami Dozo, Some geometric properties related to the fixed point theory for nonexpansive mappings, Pacific J. Math. 40 (1972), 565-573. MR 46 \#9815.

7. T. Kato, Accretive operators and nonlinear evolution equations in Banach spaces, Proc. Sympos. Pure Math., vol. 18, Part 1, Amer. Math. Soc., Providence, R. I., 1970, pp. 138-161. MR 42 \#6663.

8. S. Reich, Asymptotic behavior of contractions in Banach spaces, J. Math. Anal. Appl. 44 (1973), 57-70.

9. - Asymptotic behavior of semigroups of nonlinear contractions in Banach spaces (to appear).

10. C.-L. Yen, The range of m-dissipative sets, Bull. Amer. Math. Soc. 78 (1972), 197-199. MR 44 \#7395. ISRAEL

DEPARTMENT OF MATHEMATICAL SCIENCES, TEL AVIV UNIVERSITY, TEL AVIV,

Current address: Department of Mathematics, University of Chicago, Chicago, Illinois 60637 